\title{
Condensed Matter Physics as a Laboratory for Gravitation and Cosmology
}

\author{
Fernando Moraes \\ Laboratório de Física Teórica e Computacional \\ Departamento de Física, Universidade Federal de Pernambuco \\ 50670-901 Recife, PE, Brazil \\ E-mail: moraes@df.ufpe.br
}

Received 7 January, 2000

\begin{abstract}
The geometric language of General Relativity is not normally related to Condensed Matter (CM) Physics since it is the electromagnetic and not the gravitational interaction that dominates the physics of CM systems. What points in common would then CMP have with Cosmology and the dynamics of objects in a gravitational field? There is at least one that is very important: topological defects formed in symmetry breaking phase transitions. To explore the similarities and differences here has been a very fruitful experience for both sides. On one hand, topological defects in solids started to be described by a gravity-like theory including torsion and, on the other hand, experiments have been proposed and performed in CM systems with the purpose of testing cosmological theories. Some examples are: 1) Landau levels and the Aharonov-Bohm effect of electrons moving in a crystal containing a screw dislocation can be described in a simple way in a geometric formalism; 2) closed timelike curves have been proposed in the vicinity of vortices in superfluid Helium; 3) Kibble mechanism, for the generation of topological defects, has been experimentally verified in liquid crystals. In summary, Condensed Matter Physics with its rich diversity of systems and phenomena and of relatively easy access to experiments, appears as a laboratory for testing hypotheses of gravitational theory and cosmology involving topological defects. In this work I summarize recent results in this interface area focusing mainly in the results obtained by our research group.
\end{abstract}

\section{Introduction}

Topological defects, both in Condensed Matter Physics and in Cosmology, are formed during symmetry breaking phase transitions[1]. The analogy is so strong that, in the past few years, some "cosmological experiments" have been carried out in liquid crystals[2] and superfluid Helium[3], shading a new light on the dynamics of the defect formation process. Although quite convenient to study the dynamics of defect formation, those systems do not provide an easy experimental setting for the study of static properties of the defect background, due to the high mobility of the defects. On the other hand, topological defects in crystalline solids, with their very low mobility, are just ideal for this purpose. But, how similar are these defects to space-time defects? It is the intent of this work to briefly review these similarities and to present a summary of results on this research area obtained by our research group.

There is a formal equivalence between threedimensional gravity with torsion and the theory of defects in solids[4]. Defects in crystals are formed when the continuous translational and rotational symmetries of the liquid are broken into the discrete symmetries of the lattice during the freezing transition. They can be conceptually generated by a "cut and glue" process, known in the literature as the Volterra process[5]. This process gives a unifying view of the topological line defects. That is, take a cylinder of a continuous elastic material and make a radial cut in it, from its axis out. Displacement of the surfaces of the cut with respect to each other and subsequent glueing will generate a line defect whose core coincides with the axis. Considering cylindrical coordinates, if the displacement is:

(i) along the $z$-direction a screw dislocation is formed.

(ii) along the $r$-direction, an edge dislocation is formed.

(iii) along the $\theta$ direction, which implies the addition or removal of a wedge of material, leads to a disclination.

(iv) a combination of both (i) and (iii) it produces a dispiration.

It is clear then, that the core of such line defects are associated to geometric singularities. Here we come close to gravity theory, where geometric singularities are sources of gravitational field which, on the other hand, is described by a deformation of the space-time 
from the flat Minkowsky geometry.

\section{Geometric model for line de- fects in elastic solids}

It is well known that elastic solids with topological defects can be described by Riemann-Cartan geometry[6]. Recently, Katanaev and Volovich[4] have shown the equivalence between three-dimensional gravity with torsion and the theory of defects in solids. The defect acts as a source of a "gravitational" distortion field. The metric describing the medium surrounding the defect is then a solution to the three-dimensional EinsteinCartan equation.

Since we are discussing line defects it is convenient to imagine flat three-dimensional space as a pile of "pancakes". This way we can imagine a Volterra process for a single "pancake", and then, extend the deformation to the rest of the pile. Obviously the metric describing the space is

$$
d s^{2}=d z^{2}+d r^{2}+r^{2} d \theta^{2}
$$

in cylindrical coordinates.

A screw dislocation corresponds to a pile of cut "pancakes" like the one in Fig. 1. The displacement between the edges of the cut is the so-called Burgers vector of modulus $b$ and directed along the $z$-axis. The corresponding metric is[7]

$$
d s^{2}=\left(d z+\frac{b}{2 \pi} d \theta\right)^{2}+d r^{2}+r^{2} d \theta^{2} .
$$

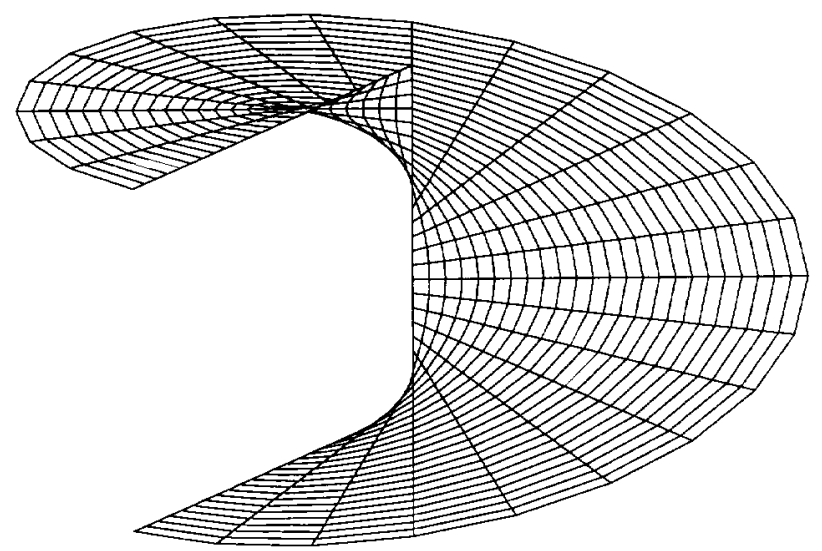

Figure 1. Screw Dislocation

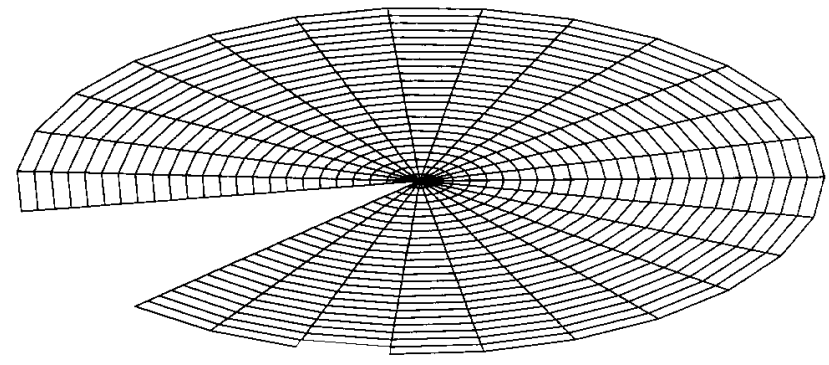

Figure 2. Disclination

Fig. 2 corresponds to the disclination. The metric of a disclinated medium, which is in fact the space section of the cosmic string metric[8], is given by

$$
d s^{2}=d z^{2}+d r^{2}+\alpha^{2} r^{2} d \theta^{2}
$$

where $\alpha$ gives a measure of the angular deficit. Since a slice of angle $2 \pi(1-\alpha)$ has been taken out of space, the total algle around the $z$-axis is now $2 \pi \alpha$.

The above metrics are in fact particular cases of the one describing the spinning chiral string space-time considered by Gal'tsov and Letelier[9] and Tod[10]

$$
d s^{2}=(d t+J d \theta)^{2}-d r^{2}-\alpha^{2} r^{2} d \theta^{2}-(d z+\beta d \theta)^{2},
$$

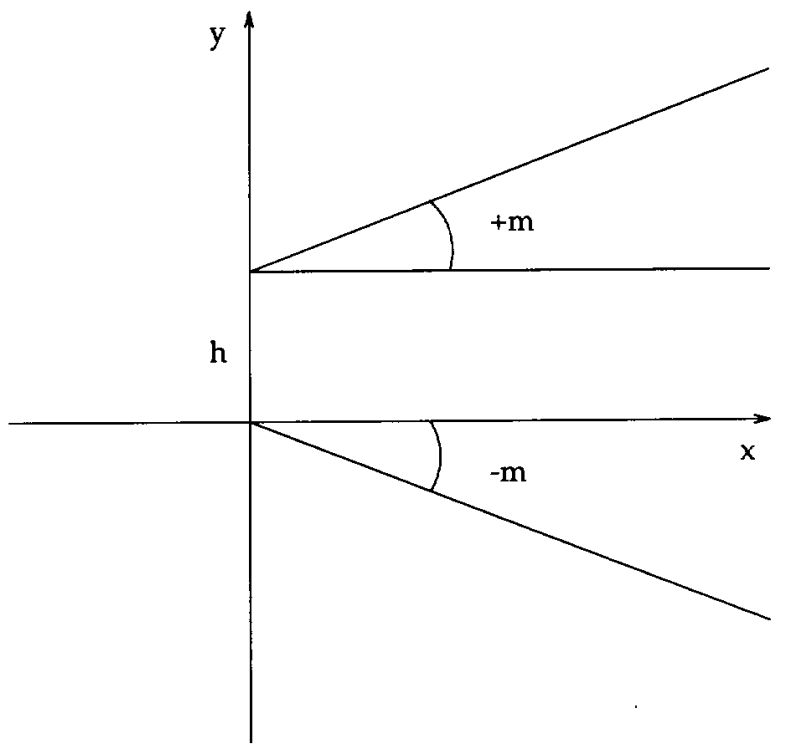

Figure 3.Edge dislocation viewed as a disclination dipole.

An edge dislocation may be formed [4] by placing a pair of opposing parallel disclinations next to each other as a dipole (Fig. 3). Each disclination is made by either removing or inserting a wedge of material. In the continuum limit, at large distances from the defect, the medium is described by the metric [4]

$$
d s^{2}=d z^{2}+\left(1+\frac{m}{2 \pi} \frac{2 h r \sin \theta-h^{2}}{r^{2}}\right)\left(d r^{2}+r^{2} d \theta^{2}\right),
$$


in cylindrical coordinates. The wedge angles are given by $\pm m$ and the disclinations are at a distance $h$ apart. The Burgers vector $\vec{b}=-m h \hat{\mathrm{x}}$ is therefore along the $\mathrm{x}$-axis and the defect itself is along the $\mathrm{z}$-axis.

Naturally, there are more defects and, consequently, more metrics. They are all listed in[7]. We will be interested in just the ones described above.

\section{Some results}

We have been mainly interested in the physical properties of crystals with defects where the defects have the passive role of providing the background for particles (electrons or holes) or fields (electromagnetic or scalar) to be around. We have also looked at similar defects in space-time. The defects impose boundary conditions on those objects leading to bound and scattering states for the particles: binding of electrons and holes to defects[12, 13], Landau levels in the presence of the defects[14, 15], localized states[16], geodesic motion[11, 17], transport phenomena[18], Aharonov-Bohm effect[19, 20, 21], quantum scattering by defects[22], the acquisition of a quantum (Berry's) phase[23]. For the fields, the boundary conditions due to the defects lead to classical and quantum effects like self-force[24, 25, 26], Casimir effect[27, 28] and corrections to physical constants[29]. Among these results I choose to comment briefly on: (a) the geodesics around an edge dislocation[11], (b) the self-force on a charged particle also near an edge dislocation[26], (c) the correction to the magnetic moment of the electron near a disclination[29] and, finally, (d) the Aharonov-Bohm effect around a screw dislocation[20, 21] .

(a) As shown in Fig. 3, an edge dislocation may be formed by removing a rectangular slice of material and glueing the loose edges. The medium with this defect is associated to the metric (5) which describes essentially a flat three-dimensional space with singular torsion on the $z$-axis. Notice the assymmetry of the metric as one go around the $z$-axis. This become quite evident when one observes the geodesics in such medium[11] (Figures 4-6). Each figure shows a bundle of geodesics, parallel to each other at infinity, approaching and being deflected by the dislocation located at the origin of the plots.

(b) The boundary conditions imposed by a topological defect on the electric field of a point charge in its neighborhood provokes a distortion of the field lines. This can be interpreted as due to image charges, just like in the case of a point charge in the presence of a ground conducting plane. In this case the interaction energy between the charge and its image is the selfenergy of the charge. Since it depends on the distance between the charge and the plane, the derivative of this energy with respect to this distance gives rise to an effective force between the charge and the plane. The same also happens for a point charge and a topological defect[26]. Fig. 7 shows a plot of the self-energy of a linear charge density parallel to an edge dislocation ( $\rho$ and $\varphi$ are the coordinates of the line of charge with respect to the defect, whose location coincides with the $z$-axis). The lack of axial symmetry of the defect is manifest in the plot. Notice that, as the charge goes around the defect, there are alternating regions of effective attraction and repulsion, respectively.

(c) Analogous to the classical field phenomenon of the self-force there are also quantum field effects due to topological defects. The Casimir effect[27, 28] being the best known example of such phenomena. A less known effect, but very important for condensed matter experiments is the correction of the magnetic moment of the electron due to a defect[29]. Quantum electrodynamics gives us

$$
\mu=\frac{e \hbar}{2 m c}\left(1+\frac{e^{2}}{2 \pi \hbar c}+\ldots\right),
$$

for the magnetic moment of the electron in empty space. The correction to the Dirac value $\mu=\frac{e \hbar}{2 m c}$ is due to the coupling of the electron to the quantized magnetic field. When the quantized electromagnetic field is submitted to the boundary conditions imposed by a topological defect the correction obviously change. For an electron bound to a negative disclination it is found[29]

$$
\frac{\delta \mu}{\mu}=\frac{\left(1-p^{2}\right) s^{2}(p) e^{6}}{288 \pi^{4} \hbar^{3} c^{3} \epsilon^{2}},
$$

where $p=1 / \alpha$. A negative disclination corresponds to $0<p<1$, therefore $\frac{\delta \mu}{\mu}>0$, confirming experimental evidence[30] that negative curvature disclinations encourage larger local moments.

(d) Here, just a brief comment on the AharonovBohm effect around a screw dislocation, which has been studied in detail in[20, 21]. The well-known original Aharonov-Bohm effect involves a charged particle moving outside a region with magnetic flux. If the flux is confined to a string, it can be described, in the KaluzaKlein approach, as the five-dimensional metric

$$
d s^{2}=d t^{2}-d z^{2}-d r^{2}-r^{2} d \theta^{2}-\left(d x^{5}+\frac{\Phi}{2 \pi} d \theta\right)^{2},
$$

where $\Phi$ is the magnetic flux and $x^{5}$ the fifth dimension. Now, compare this metric with the four-dimensional metric of a screw dislocation (see Equation (2)

$$
d s^{2}=d t^{2}-\left(d z+\frac{b}{2 \pi} d \theta\right)^{2}-d r^{2}-r^{2} d \theta^{2} .
$$

Notice that the Burgers vector $b$ plays the same role as the magnetic flux $\Phi$ making it clear that the AharonovBohm effect should appear for a particle moving in the presence of a screw dislocation. Indeed, the Schrödinger equation will look the same in either background ( $\Phi$ being replaced by $b$ ) being exactly Aharonov-Bohm's equation[19]. 


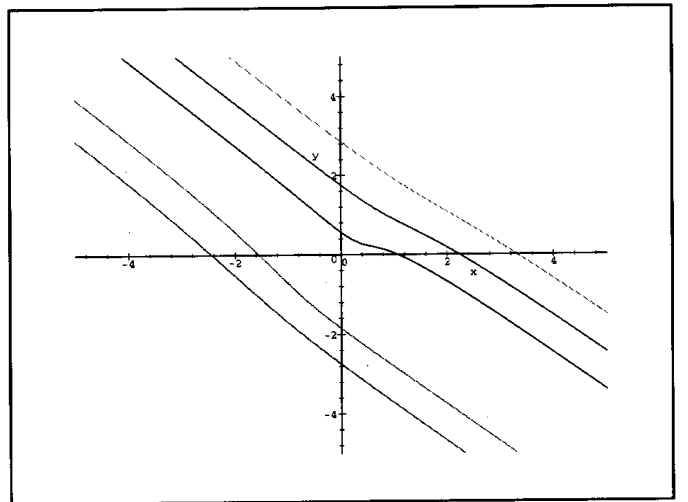

Figure 4. Geodesics around edge dislocation (1).

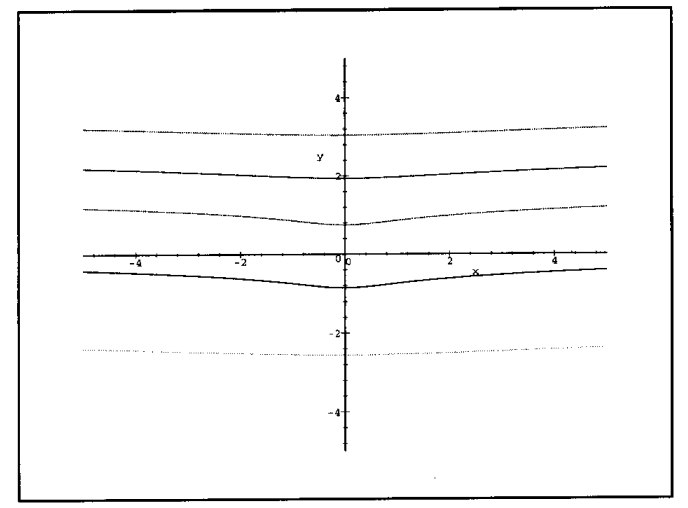

Figure 5. Geodesics around edge dislocation (2).

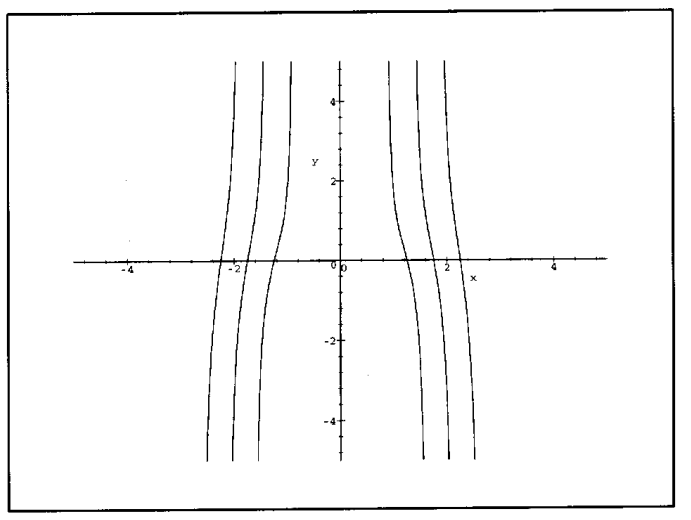

Figure 6. Geodesics around edge dislocation (3).

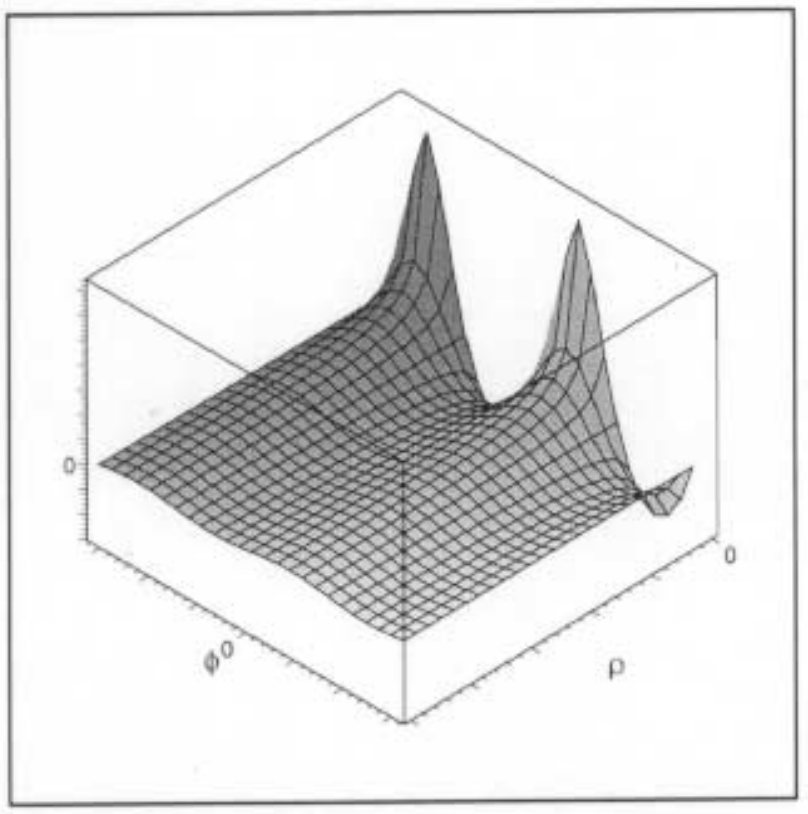

Figure 7. Self-energy of a linear charge density parallel to an edge dislocation.

\section{Other systems}

Topological defects appear in nature as consequence of symmetry-breaking phase transitions. An important issue in cosmology is whether the observed structure of the universe contains relics of topological defects formed as the early universe cooled. What is the defect density after a phase transition? In 1976 T. W. B. Kibble[31] proposed a statistical procedure for calculating the probability of string formation in the phase transitions in the early universe. In 1991 Chuang and coworkers[32] and in 1994 Bowick et al. made experimental verifications of the Kibble mechanism for string formation in liquid crystals. Since 1994 different experimental groups[3] have tested Kibble's mechanism in superfluid $\mathrm{He}^{4}$.

More interesting than superfluid $\mathrm{He}^{4}$ are the superfluid phases of $\mathrm{He}^{3}$ which are quantum liquids with interacting fermionic and bosonic fields. Its rich structure gives rise to a number of analogues of cosmological defects[33]: 1) the dysgiration, that simulates the extremely massive cosmic string; 2 ) the singular vortex, which is analogous to the rotating cosmic string, 3) the continous or ATC vortex, whose motion causes "momentogenesis", which is the analogue of baryogenesis in the early universe; 4) planar solitons, that have event horizons similar to rotating black and white holes; 5) symmetric vortices (in a thin film), which admit the existence of closed timelike curves through which only superfluid clusters of anti- $\mathrm{He}^{3}$ atoms can travel and violate causality; 6) moving domain walls that can generate Hawking radiation; and so on. 
There are still the superconductors with their vortices; glasses with their curved space description that require disclinations and perhaps monopoles; magnets with their disclinations and domain walls...

\section{Conclusion}

Condensed Matter Physics has much benefited from tools and ideas from gravitation and cosmology and pays that back by offering a laboratory for testing some cosmological or gravitational hypotheses.

\section{Acknowledgments}

My warmest thanks go to my numerous collaborators (see references [12-28]) who made this work come out of the vacuum. This work was partially supported by FACEPE, CNPq and FINEP through its PRONEX program.

\section{References}

[1] Tammay Vachaspati, Phys. Rev. D 44, 3723 (1991).

[2] Mark J. Bowick et al., Science 263, 943 (1994).

[3] W. H. Zurek, Phys. Rep. 276, 177 (1996).

[4] M. O. Katanaev and I. V. Volovich, Ann. Phys. (NY) 216, 1 (1992).

[5] M. Kleman, Points, Lignes, Parois: dans les fluides anisotropes et les solides cristallins (Edition de Physique, France 1977).

[6] E. Kröner, Continuum theory of defects, in: Les Houches, Session XXXV, 1980 - Physics of Defects, eds. R. Balian et al. (North-Holland, Amsterdam, 1981) pp. 282-315.

[7] Roland A. Puntigam and Harald H. Soleng, Class. Quantum Grav. 14, 1129 (1997).

[8] A. Vilenkin, Phys. Rev. D 23, 852 (1981).

[9] D. V. Gal'tsov and P. S. Letelier, Phys. Rev. D 47, 4273 (1993).

[10] K. P. Tod, Class. Quantum Grav. 11, 1331 (1994).

[11] Fernando Moraes, Phys. Lett. A 214, 189 (1996).

[12] Claudio Furtado and Fernando Moraes, Phys. Lett. A 188, 394 (1994).

[13] Claudio Furtado and Fernando Moraes, Harmonic Oscillator Interacting with Conical Singularities, submitted to publication.
[14] C. Furtado et al., Phys. Lett. A 195, 90 (1994).

[15] Claudio Furtado and Fernando Moraes, Europhys. Lett. 45, 279 (1999).

[16] Sérgio Azevedo, Claudio Furtado and Fernando Moraes, Physica Status Solidi (b), 207, 387 (1998).

[17] A. de Padua, Fernando Parisio-Filho and Fernando Moraes, Phys. Lett. A 238, 153 (1998).

[18] A.P. Balachandran, Varghese John, Arshad Momen and Fernando Moraes, Int. J. Mod. Phys. A 13, 841 (1998).

[19] Sérgio Azevedo and Fernando Moraes, Phys. Lett. A 246, 374 (1998).

[20] Claudio Furtado, Fernando Moraes and V. B. Bezerra, Phys. Rev. D 59, 107504 (1999).

[21] Claudio Furtado, Fernando Moraes and V. B. Bezerra, Loop Variables and Aharonov-Bohm effect in KaluzaKlein theory, Accepted for publication in Mod. Phys. Lett. A (2000).

[22] Sérgio Azevedo and Fernando Moraes, Two-dimensional scattering by disclinations in monolayer graphite, submitted to publication.

[23] Claudio Furtado, V. B. Bezerra and Fernando Moraes, Berry's Quantum Phase in Dislocated Media, submitted to publication.

[24] E.R. Bezerra de Mello, V.B. Bezerra, C. Furtado and F. Moraes, Phys. Rev. D 51, 7140 (1995).

[25] Claudio Furtado and Fernando Moraes, Class. Quantum Grav. 14, 3425 (1997).

[26] A. M. de M. Carvalho, Claudio Furtado and Fernando Moraes, Self-forces on electric and magnetic linear sources in the presence of a torsional defect, submitted to publication.

[27] Fernando Moraes, Phys. Lett. A 204, 399 (1995).

[28] Ivan Pontual and Fernando Moraes, Phil. Mag. A 78, 1073 (1998).

[29] Fernando Moraes, Mod. Phys. Lett. A 10, 2335 (1995).

[30] L. H. Bennet and R. E. Watson, Phys. Rev. B 35, 845 (1987).

[31] T. W. B. Kibble, J. Phys. A Gen. Phys. 9, 1387 (1976).

[32] I. Chuang, N. Turok and B. Yurke, Phys. Rev. Lett. 66, $2472(1991)$

[33] G. E. Volovik, P. Natl. Acad. Sci. USA 96, 6042 (1999) and references therein 